\title{
PyWD2015 - A new GUI for the Wilson-Devinney code
}

\author{
O. Güzel and O. Özdarcan \\ Ege University, Faculty of Science, Astronomy and Space Sciences \\ Department, 35100 Bornova İzmir, TÜRKIYYE (E-mail: \\ ozanguzel35@outlook.com)
}

Received: October 8, 2019; Accepted: November 5, 2019

\begin{abstract}
A new, modern graphical user interface (GUI) for the 2015 version of the Wilson-Devinney (WD) code is developed. PyWD2015 is written in Python 2.7 and uses the Qt4 interface framework. At its core, the GUI generates lcin and dcin files from user inputs and sends them to WD, then reads and visualises the output in a user friendly way. It also includes some useful tools for the user, which makes technical aspects of the modelling process significantly easier. While multiple sky surveys and space missions generate, reduce and categorize large amounts of observational data, it's up to dedicated studies to analyse peculiar or anomalous systems and make further progress in the field of physics of eclipsing binaries. We believe PyWD2015 will be a great "dedicated study" suite for such systems.
\end{abstract}

Key words: binaries: eclipsing - methods: data analysis

\section{Introduction}

In the age of space telescopes and large surveys, automated data pipelines play a major role. While these automated pipelines are very efficient for sorting and cataloguing large amounts of data, anomalous and interesting systems need individual attention and manual analyses. These analyses often require physical modelling of the system in question, specifically in the case of eclipsing binaries.

The Wilson-Devinney code (Wilson \& Devinney, 1971) for eclipsing binary modelling is generally considered a standard in the field. The code is proven reliable and carries a long legacy of outputting correct science, therefore it is suited well for performing detailed analysis on various types of interesting eclipsing binaries.

However, it is not free from caveats. It requires strictly formatted input files for operation and lacks any visualisation of output results. Creating and formatting input files and reading outputs have a tendency to become technical hurdles, which slows down the whole analysis process. In order to avoid these hurdles and provide some additional physics, the PHOEBE 1.0 project (Prša \& Zwitter, 2005) was developed by adopting ithe WD code as backend. However, 
PHOEBE 1.0 is mainly based on ithe 2003 version of the WD code, while the latest version is 2015 (Wilson \& Van Hamme, 2014).

In this contribution, a GUI for the latest (2015) version of the WD code, named "PyWD2015," is presented. The GUI covers almost all features of the code with some additional tools.

\section{Overview}

PyWD2015 is, at its core, a "GUI wrapper" for the WD code. It does not abstract or redefines any WD functionality, but merely provides a convenient interface for inputting parameters and running the DC and LC programs. When the user runs any WD tool, the GUI takes input parameters and observations, formats them automatically, writes them onto disk in an input file and finally executes the LC or DC binary. Then, after WD completes its run, the GUI reads the output file and visualises the outputs in a user-friendly and convenient way.

PyWD2015 provides a main menu for inputting global parameters for a system, but has separate windows for LC and DC inputs. Each LC functionality has its own separate window but they share the same LC specific parameters. DC, on the other hand, has a single window which runs the DC program and shows optimized parameters accompanied with an embedded plot window for visualising results. Some of these windows can be seen in Fig. 1. The only WD feature that is not supported by PyWD2015 is the "subset" functionality of DC, but that is planned to be included in a future release.

In addition to providing a GUI wrapper for WD, PyWD2015 also has some user-friendly features:

- Most of the tooltips of labels are filled with the excerpts taken from the WD manual, ${ }^{1}$ and some of them have alternative, longer explanations.

- The "Star Positions" window of the LC tool has the ability to draw critical Roche potentials of the system. This has been written into the GUI and does not depend on WD. Calculations are adopted from Kopal (1959) and Kallrath \& Milone (2009).

- The DC tab has a "Solution Explorer" window, which tracks subsequent DC iteration results. Each parameter can be plotted with its standard deviation as an error bar to help the evaluation of solution progress.

- The DC window also has an "external iteration" variable, which is different from the WD parameter NITER. This variable tells the number of times the GUI needs to iterate the DC solution externally, thus enabling the "Solution Explorer" window to track solution progress. After each iteration, the DC window automatically updates the outputs and copies results to input parameters.

${ }_{1}$ ftp://ftp.astro.ufl.edu/pub/wilson/lcdc2015/ebdoc.6jun2016.pdf 


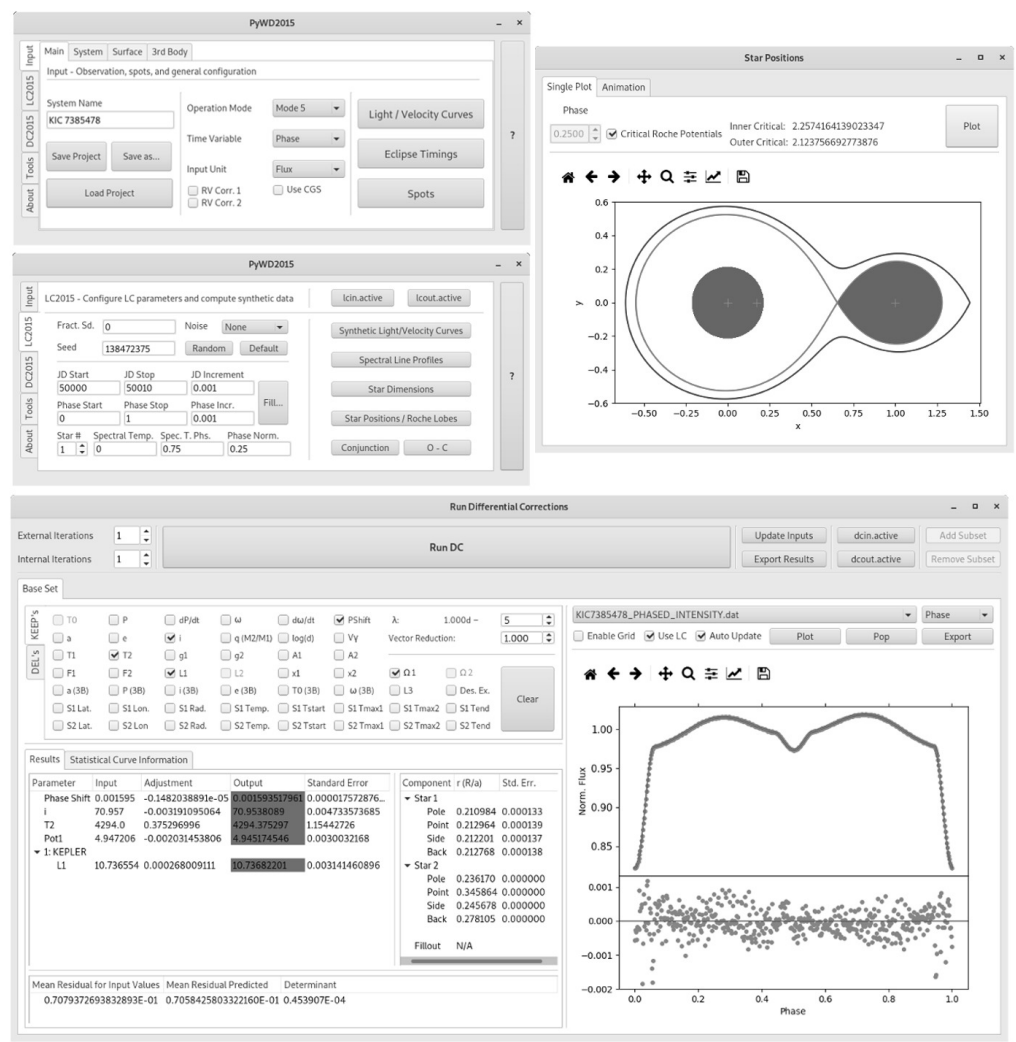

Figure 1. Representative windows of PyWD2015. Top left are the main and LC windows. The "Star Positions" window is at the top right. Below is the DC window.

- GUI also has some numerous colour-temperature calibrations adopted from various references (Gray, 2005; Drilling \& Landolt, 2000; Flower, 1996; Popper, 1980; Tokunaga, 2000). These are implemented by using 7 th order polynomials (except for Gray (2005), who adopts 4th order polynomial for cool stars and 5 th order polynomial for hot stars).

- Additional simple and useful tools, such as time (JD-UT) conversion and dimensionless potential calculations based on mass ratio, fractional radius, rotation parameter and instant separation, are available.

- The GUI can save and load project files to enable project management.

Some external Python libraries were used in PyWD2015. Matplotlib library (Hunter, 2007) was used for plot widgets. Numpy (Oliphant, 2015) and SciPy (Jones et al., 2001) libraries were used for numerical calculations. 


\section{Conclusion}

In this contribution, a GUI for the 2015 version of the WD code is presented. The GUI is a wrapper for the WD code with user friendly features. It covers almost every WD functionality with added visualisation tools. The code and user manual can be found on GitHub. ${ }^{2}$

Acknowledgements. The authors are thankful to Dr. Robert E. Wilson and Dr. Walter Van Hamme for their permissions regarding the redistribution of WD code and limb darkening tables along with PyWD2015. The authors also thanks Dr. Dirk Terrel for his constructive comments and suggestions on PyWD2015.

\section{References}

Drilling, J. S. \& Landolt, A. U. 2000, Normal Stars, Allen's Astrophysical Quantities, 4th Edition, ed. A. N. Cox, Springer-Verlag, Berlin, p. 381

Flower, P. J., Transformations from Theoretical Hertzsprung-Russell Diagrams to Color-Magnitude Diagrams: Effective Temperatures, B-V Colors, and Bolometric Corrections. 1996, Astrophys. J., 469, 355, DOI: 10.1086/177785

Gray, D. F. 2005, The Observation and Analysis of Stellar Photospheres, 3rd Edition, Cambridge University Press, Cambridge

Hunter, J. D., Matplotlib: A 2D graphics environment. 2007, Computing in Science 8 Engineering, 9, 90, DOI: 10.1109/MCSE.2007.55

Jones, E., Oliphant, T., Peterson, P., et al. 2001, SciPy: Open source scientific tools for Python

Kallrath, J. \& Milone, E. F. 2009, Eclipsing Binary Stars: Modeling and Analysis, Springer, New York

Kopal, Z. 1959, Close binary systems, Chapman \& Hall, London

Oliphant, T. E. 2015, Guide to NumPy, 2nd Edition, CreateSpace Independent Publishing Platform, USA

Popper, D. M., Stellar masses. 1980, Ann. Rev. Astron. Astrophys., 18, 115, DOI: 10.1146/annurev.aa.18.090180.000555

Prša, A. \& Zwitter, T., A Computational Guide to Physics of Eclipsing Binaries. I. Demonstrations and Perspectives. 2005, Astrophys. J., 628, 426, DOI: $10.1086 / 430591$

Tokunaga, A. T. 2000, Infrared Astronomy, Allen's Astrophysical Quantities, 4th Edition, ed. A. N. Cox, Springer-Verlag, Berlin, p. 143

Wilson, R. E. \& Devinney, E. J., Realization of Accurate Close-Binary Light Curves: Application to MR Cygni. 1971, Astrophys. J., 166, 605, DOI: 10.1086/150986

Wilson, R. E. \& Van Hamme, W., Unification of Binary Star Ephemeris Solutions. 2014, Astrophys. J., 780, 151, DOI: 10.1088/0004-637X/780/2/151

${ }^{2}$ https://github.com/Varnani/PyWD2015 